\title{
All Optical Communication Filter Based on Photonic Crystal Structure
}

\author{
Hamed Alipour-Banaei, Farhad Mehdizadeh, and Behnaz Amini
}

\begin{abstract}
In this paper we proposed a novel structure for designing all optical filter based on photonic crystal structure. For designing the proposed filter we simply employed a point defect localized between input and output waveguides as wavelength selecting part of the filter. The initial form of this filter is capable of selecting optical waves at $\cdot=1554.2 \mathrm{~nm}$, the transmission efficiency of the filter is $100 \%$. In designing and studying the optical properties of the filter we used plane wave expansion and finite difference time domain methods. After designing the filter we studied the impact of different parameters on the filtering behavior of the structure. The total footprint of the filter is less than $76 \bigcirc \mathbf{m}^{2}$. Simplicity of design and ultra-compact dimensions are the most significant characteristics of our filter.
\end{abstract}

Index Terms-Defect, FDTD, optical filter, photonic crystal.

\section{INTRODUCTION}

Currently optical communication networks and optical systems are the best solutions for meeting the ever increasing demand of human kind for fast and high band width communication links, required for sending and receiving the required data throughout the world. For realizing all optical communication networks we require all optical devices such as optical filters, optical demultiplexers and optical switches. One crucial challenge in designing ultra-compact optical devices is the poor confinement of light in small spaces. This challenge has been solved through employing photonic crystals (PhCs) [1]. PhCs have a special frequency (wavelength) range in which the propagation of optical waves inside these artificial structures is forbidden. This special range is called photonic band gap (PBG) [2], [3]. Optical filters play a crucial role in optical communication networks. They used for choosing the desired wavelength and also for separating the very closely spaced optical channels in wavelength division multiplexing (WDM) applications [4]. If we add a defect layer in a $1 \mathrm{DPhC}$ we can realize a narrow band transmission filter [5]. In these structures the pass band is located in the PBG region of $1 \mathrm{D}$ PhC. by replacing the defect layer by a photonic quantum well [6] we can have a multichannel filter. Another way of realizing multichannel filter based on $1 \mathrm{DPhC}$ is replacing dielectric layers by superconducting $\mathrm{PhC}$, in these structures there is no defect layer [7]. Thue-Morse multilayers are other structures proposed for designing optical filters [8]. All of these aforementioned filters are based on 1D PhCs. 1D PhCs do not have complete band gaps so their optical behavior is very

Manuscript received July 1, 2015; revised September 21, 2015.

The authors are with the Department of Electronics, Tabriz Branch, Islamic Azad University, Tabriz, Iran (e-mail: alipour@iaut.ac.ir). sensitive upon the incident angle of the input light.

Other kind of optical filters are designed based on 2D PhCs. 2D PhCs due to their complete band gaps are not sensitive upon the incident angle of input light. Different mechanisms have been proposed for designing optical filters based on $2 \mathrm{DPhCs}$. One way of realizing optical filter in $2 \mathrm{DPhCs}$ is putting a resonant cavity between input and output waveguides [9], [10]. It has been shown that the resonant wavelength of the cavity can be controlled by changing the effective length of the resonant cavity. Using quasi crystal structures are other way of realizing optical filters based on 2DPhCs [11], [12]. The most common mechanism used for designing $2 \mathrm{DPhC}$ based filters is ring resonators [13]-[16]. Different kinds of ring resonators have been proposed most recently. In these structures the resonant wavelength depends on the refractive index and dimensions of the resonant ring [17].

In this paper we are going to propose a novel structure for designing all optical filter. In this paper we used a point defect located between input and output waveguides as wavelength selecting mechanism. High transmission efficiency, ultra-compact dimension and simplicity of design are the most significant advantages of our filter. Our results show that by increasing the radius of defect and also the radius of air pores a blue shift will occur in the output wavelengths of the filter, however increasing the refractive index of the dielectric substrate results in a red shift in the output wavelengths of the filter.

\section{THEORETICAL MODELING AND METHODS}

The fundamental platform used for designing the proposed filter is a hexagonal lattice of air pores created in a dielectric substrate. The number of air pores in $\mathrm{x}$ and $\mathrm{y}$ directions are 21 and 15 respectively. The effective refractive index of dielectric material is 2.73 . The radius of air pores is $190 \mathrm{~nm}$ and the lattice constant of the structure is $530 \mathrm{~nm}$. Before designing the filter we have to calculate the band structure of the fundamental $\mathrm{PhC}$ and extract its $\mathrm{PBG}$ region. For this purpose we employed Bandsolve simulation tool of Rsoft photonic CAD software, which calculates the band structure of periodic structures based on plane wave expansion (PWE) method [18].

The band structure diagram for this $\mathrm{PhC}$ with aforementioned values for refractive index of dielectric material, radius of air pores and lattice constant is shown in Fig. 1. According Fig. 1 this structure has one PBG in TE mode that is shown by dark area in the band structure of the $\mathrm{PhC}$. TE mode is defined as a mode in which light polarization is perpendicular to air pores. There is no PBG for TM mode so all the simulations will be performed in TE 
mode. We observe from Fig. 1 that PBG is in the range of $0.282<a / \lambda<0.395$ or in other words $1341 \mathrm{~nm}<\lambda<1879 \mathrm{~nm}$.

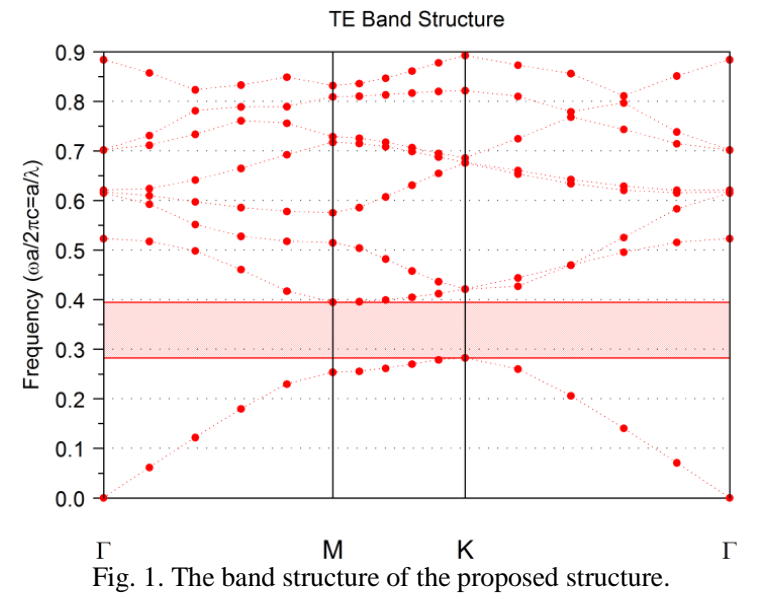

Our proposed filter is composed of three main parts: 2 line defects as input waveguide and output waveguide and a point defect located between the waveguides. For creating the point defect, we reduced the radius of one cell between the waveguides, this change in the radius of one cell between the waveguides creates resonant modes between two waveguides, so optical waves at certain wavelengths can be transferred form input waveguide into output waveguide, through the resonant defect. The schematic diagram of the proposed filter is shown in Fig. 2. The point defect is shown with dark blue color, whose radius is $R=76 \mathrm{~nm}$.

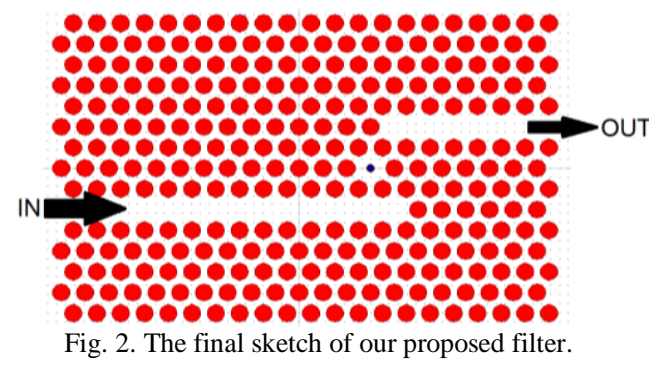

\section{Simulation AND RESUlts}

After finalizing the design process of the filter we have to investigate the optical behavior of the proposed filter. For this purpose we used Fullwave simulation tool of Rsoft photonic CAD software for testing our proposed structure. Fullwave studies the propagation of light inside $\mathrm{PhC}$ based devices based on finite difference time domain (FDTD) method [19]. We used Perfectly Matched Layer (PML) boundary condition for simulating our proposed demultiplexers [20]. We choose the PML width surrounding our structures to be $500 \mathrm{~nm}$. For accurate modeling of the filter we need 3D simulation, but it requires great amount of run time and very powerful computer. So we used effective index approximation method of $\mathrm{PhCs}$ for satisfying this requirement and with this approximation we reduce the $3 \mathrm{D}$ simulations to $2 \mathrm{D}$ simulations [21].

Grid sizes $(\Delta x$ and $\Delta y)$ in FDTD parameters are chosen to be a/16 which equals $33 \mathrm{~nm}$. Due to stability considerations of the simulation the time step $\Delta t$ should satisfy the $\left(\Delta t \leq 1 / c \sqrt{(1 / \Delta x)^{2}+(1 / \Delta z)^{2}}\right)$ where $\mathrm{c}$ is the velocity of light in free space [22]. Following the above-mentioned rule for time step, $\Delta t=0.021 \mathrm{~ns}$ was chosen. The output spectrum of the filter has been obtained and shown in Fig. 3. Fig. 4 shows that are structure works as an optical filter which only selects the wavelength of $1554.2 \mathrm{~nm}$, the bandwidth $(\Delta \lambda)$ is $2.2 \mathrm{~nm}$ and the Q-factor $\left(Q=\lambda_{0} / \Delta \lambda\right)$ is 706 . The transmission efficiency of this filter is $100 \%$. The distribution of optical waves at $\lambda=1554.2 \mathrm{~nm}$ inside the filter is shown in Fig. 4, as we can see at this wavelength the input waves will couple to the output waveguide through the resonant defect. At the following we are going to investigate the effect of different parameters on the filtering behavior of the proposed filter.

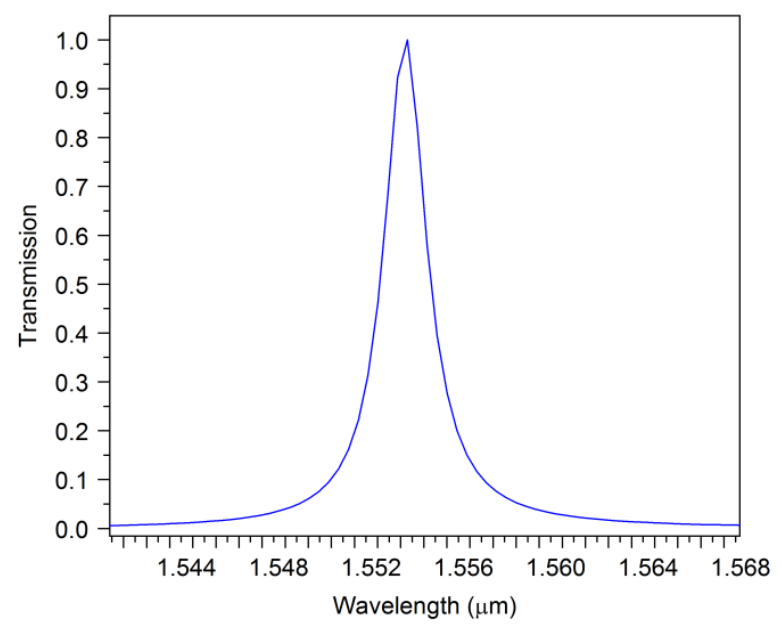

Fig. 3. The output spectrum of our proposed filter.

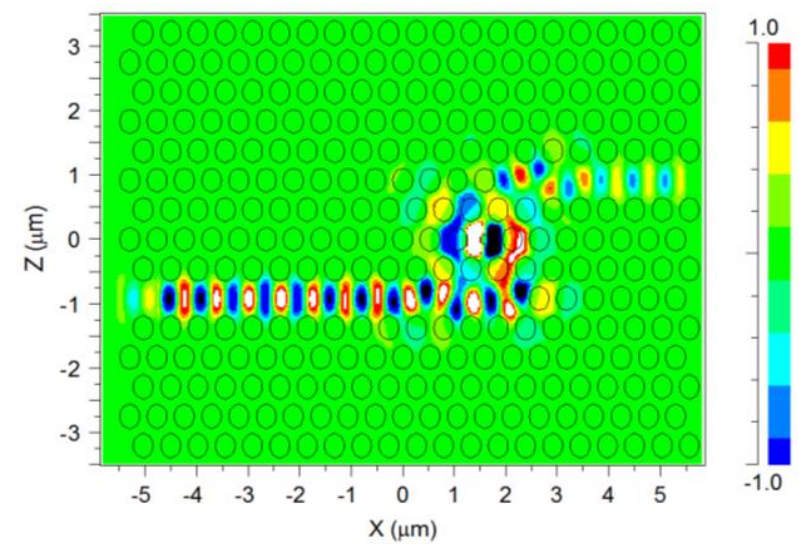

Fig. 4. The distribution of optical waves inside the filter at $\lambda=1554.2 \mathrm{~nm}$.

The output spectra of the structure for different values of $\mathrm{R}$ -radius of defect- is shown in Fig. 5. As shown in Fig. 5 by increasing $\mathrm{R}$, we have a blue shift in output wavelengths, because the resonant wavelengths decrease. Such that for $R=60,65,70,75$, and $80 \mathrm{~nm}$ the output wavelengths are $\lambda=1579.1 \mathrm{~nm}, 1571.9 \mathrm{~nm}, 1563.7 \mathrm{~nm}, 1554.2 \mathrm{~nm}$ and 1544.7 $\mathrm{nm}$ respectively.

The detailed specifications of the output wavelengths for different values of $\mathrm{R}$ are listed in Table $\mathrm{I}$.

TABLE I: SigNIFICANT PARAMETERS OF THE PROPOSED FILTER FOR

\begin{tabular}{lllll}
\multicolumn{5}{c}{ DIFFERENT VALUES OF $R$} \\
\hline$R$ & $\lambda(\mathrm{nm})$ & $\Delta \lambda(\mathrm{nm})$ & $Q$ & T. E. ${ }^{*}(\%)$ \\
\hline 60 & 1579.1 & 2.5 & 631 & 90 \\
65 & 1571.9 & 2.5 & 628 & 93 \\
70 & 1563.7 & 2.3 & 679 & 97 \\
75 & 1554.2 & 2.2 & 706 & 100 \\
80 & 1544.7 & 2.2 & 701 & 98 \\
\hline
\end{tabular}

*Transmission Efficiency 


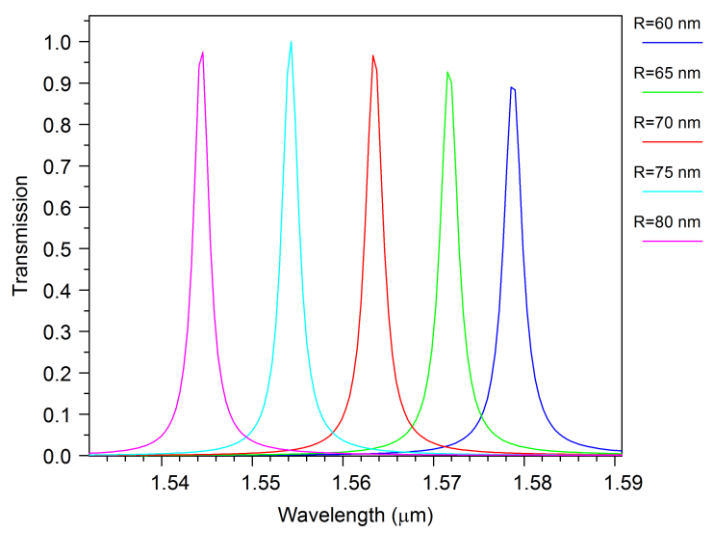

Fig. 5. The output spectra of the proposed filter for different values of $R$.

The output spectra of the structure for different values of $r$ -radius of air pores- is shown in Fig. 6. As shown in Fig. 6 by increasing $r$, we have a blue shift in output wavelengths, because the resonant wavelengths decrease. Such that for $r=188,189,190,191$, and $192 \mathrm{~nm}$ the output wavelengths are $\lambda=1567 \mathrm{~nm}, 1562 \mathrm{~nm}, 1557.6 \mathrm{~nm}, 1553.2 \mathrm{~nm}$ and $1548.7 \mathrm{~nm}$ respectively.

The detailed specifications of the output wavelengths for different values of $r$ are listed in Table II.

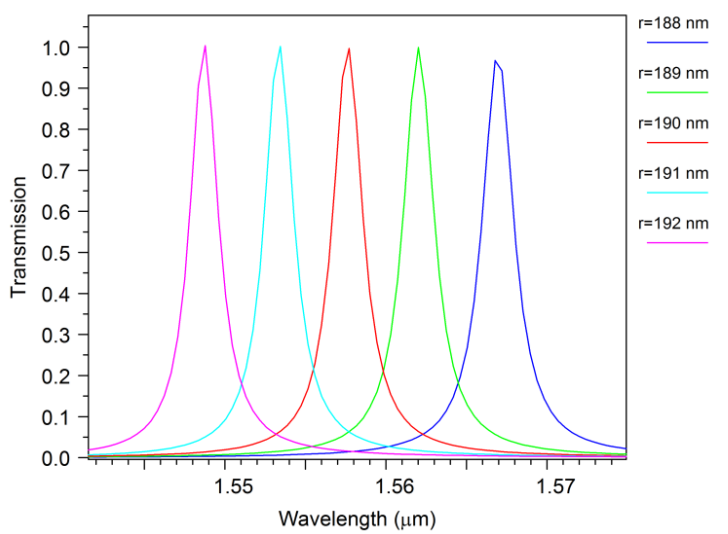

Fig. 6. The output spectra of the proposed filter for different values of $r$.

TABLE II: SigNIFICANT PARAMETERS OF THE PROPOSED FILTER FOR

\begin{tabular}{lllll}
\multicolumn{5}{c}{ DIFFERENT VALUES OF $R$} \\
\hline$r$ & $\lambda(\mathrm{nm})$ & $\Delta \lambda(\mathrm{nm})$ & $Q$ & T. E. ${ }^{*}(\%)$ \\
\hline 188 & 1567 & 2.4 & 681 & 96 \\
189 & 1562 & 2.3 & 679 & 100 \\
190 & 1557.6 & 2.2 & 708 & 99 \\
191 & 1553.4 & 2.2 & 706 & 100 \\
192 & 1548.7 & 2.2 & 703 & 100 \\
\hline \multicolumn{5}{r}{ Transmission Efficiency }
\end{tabular}

Finally, the output spectra of the structure for different values of $\mathrm{n}$-refractive index of dielectric material- is shown in Fig. 7. As shown in Fig. 7 by increasing $n$, we have a red shift in output wavelengths, because the resonant wavelengths increase. Such that for $n=2.71,2.72,2.73,2.74$, and 2.75 the output wavelengths are $\lambda=1542.3 \mathrm{~nm}, 1547 \mathrm{~nm}$, $1552 \mathrm{~nm}, 1556.6 \mathrm{~nm}$ and $1561.4 \mathrm{~nm}$ respectively. The detailed specifications of the output wavelengths for different values of $\mathrm{n}$ are listed in Table III.

The red shift and blue shift has been occurred. By increasing the radius of the cells according to Table II, the wavelength shift could be seen from $1548.7 \mathrm{~nm}$ to $1567 \mathrm{~nm}$, also the amount of blue shift and red shift tuned by the size of each defect cells. In addition by changing the refractive indices the wavelengths are shifted.

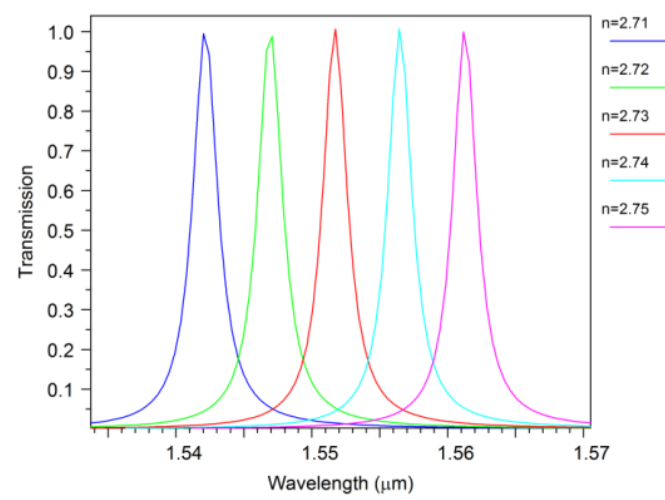

Fig. 7. The output spectra of the proposed filter for different values of $n$.

TABLE III: SIGNIFICANT PARAMETERS OF THE PROPOSED FILTER FOR DIFFERENT VALUES OF $N$

\begin{tabular}{|c|c|c|c|c|}
\hline $\mathrm{n}$ & $\lambda(\mathrm{nm})$ & $\Delta \lambda(\mathrm{nm})$ & Q & T. E. ${ }^{*}(\%)$ \\
\hline 2.71 & 1542.3 & 2.1 & 734 & 99 \\
\hline 2.72 & 1547 & 2 & 773 & 98 \\
\hline 2.73 & 1552 & 2 & 776 & 100 \\
\hline 2.74 & 1556.6 & 2.2 & 707 & 100 \\
\hline 2.75 & 1561.4 & 2.3 & 673 & 100 \\
\hline
\end{tabular}

${ }^{*}$ Transmission Efficiency

\section{CONCLUSION}

In this paper employing a point defect inside a $2 \mathrm{D}$ photonic crystal we proposed an optical filter. Using numerical methods such as PWE and FDTD methods we obtained the optical properties of our proposed structure and investigated the effect of different parameters on the output wavelength of the filter. Our results show that by increasing the radius of defect and also the radius of air pores a blue shift will occur in the output wavelengths of the filter, however increasing the refractive index of the dielectric substrate results in a red shift in the output wavelengths of the filter. The total footprint of the filter is less than $76 \mu \mathrm{m}^{2}$, this shows that our filter is compact enough to be used in all optical integrated circuits. Simplicity of design is the other advantage of our proposed structure.

\section{REFERENCES}

[1] S. John, "Strong localization of photons in certain disordered dielectric superlattices," Physical Review Letters, vol. 58, no. 23, pp. 2486-2489, 1987.

[2] B. Rezaei and M. Kalafi, "Engineering absolute band gap in anisotropic hexagonal photonic crystals," Optics Communications, vol. 266, pp. 159-163, 2006.

[3] F. Mehdizadeh and H. Alipour-Banaei, "Band gap management in two dimensional photonic crystal thue-morse structures," Journal of Optical Communications, vol. 34, pp. 61-65, 2013.

[4] H. J. R. Dutton, Understanding Optical Communications, IBM Corporation, 1998

[5] C. J. Wu and Z. H. Wang, "Properties of defect modes in one-dimensional photonic crystals," Progress in Electromagnetic Research (PIER), vol. 103, pp. 169-184, 2010.

[6] F. Qiao, C. Zhang, J. Wan, and J. Zi, "Photonic quantum-well structures: Multiple channeled filtering phenomena," Applied Physics Letters, vol. 77, pp. 3698-3701, 2000.

[7] W. H. Lin, C. J. Wu, T. J. Yang, and S. J. Chang, "Terahertz multichanneled filter in a superconducting photonic crystal," Optics Express, vol. 18, pp. 27155-27166, 2010.

[8] W. J. Hsueh, S. J. Wun, Z. J. Lin, and Y. H. Cheng, "Features of the perfect transmission in Thue - Morse dielectric multilayers," Journal of Optical Society of America B, vol. 28, pp. 2584-2591, 2011.

[9] H. Alipour-Banaei and F. Mehdizadeh. (2012). Significant role of photonic crystal resonant cavities in WDM and DWDM communication tunable filters. Optik. [Online]. Available: http://dx.doi.org/10.1016/j.ijleo.2012.07.029 
[10] H. Alipour-Banaei, M. Hassangholizadeh-Kashtiban, and F. Mehdizadeh. (2013). WDM and DWDM optical filter based on 2D photonic crystal Thue-Morse structure. Optik. [Online]. Available: http://dx.doi.org/10.1016/j.ijleo.2013.03.027

[11] A. Rostami, A. Haddadpour, F. Nazari and H. Alipour-Banaei, "Proposal for an ultracompact tunable wavelength-division-multiplexing optical filter based on quasi-2D photonic crystals," Iop- J. Opt., vol. 12, p. 015405, 2010.

[12] H. P. Bazargani, "Proposal for a 4-channel all optical demultiplexer using 12 -fold photonic crystal quasicrystal," Optics Communication, vol. 685 , pp. 1848-1850, 2012

[13] M. Djavid, A. Ghaffari, F. Monifi, and M. S. Abrishamian, "T-shaped channel-drop filters using photonic crystal ring resonators," Physica E, vol. 40, pp. 3151-3154, 2008.

[14] M. Djavid and M. S. Abrishamian, "Multi-channel drop filters using photonic crystal ring resonators," Optik, vol. 123, pp. 167-170, 2011.

[15] A. Taalbi, G. Bassou, and M. Y. Mahmoud, New design of channel drop filters based on photonic crystal ring resonators," Optik, 2012.

[16] M. Y. Mahmoud, G. Bassou, A. Taalbi, and Z. M. Chekroun, "Optical channel drop filter based on photonic crystal ring resonators," Optics Communications, vol. 285, pp. 368-372, 2012.

[17] F. Mehdizadeh, H. Alipour-Banaei, and S. Serajmohammadi, "Channel-Drop filter based on a photonic crystal ring resonator," $J$. Opt., vol. 15, 2013

[18] S. G. Johnson and J. D. Joannopoulos, "Block-iterative frequency-domain methods for Maxwell's equations in a plane wave basis," Opt. Express, vol. 8, pp. 173-190, 2001.
[19] S. D. Gedney, Introduction to Finite-Difference Time-Domain (FDTD Method for Electromagnetics, Lexington KY: Morgan \& Claypool, 2010.

[20] A. Taflove and S. C. Hegnese, Computational Electrodynamics: The Finite-Difference Time-Domain Method, Boston, MA: Artech House, 1998.

[21] M. Qiu, "Effective index method for heterostructure-slab-wave-guide-based two-dimensional photonic crystals," Appl. Phys. Lett, vol. 81, pp. 1163-1165, 2002

[22] S. Chen, B. Mulgrew, and P. M. Grant, "A clustering technique for digital communications channel equalization using radial basis function networks," IEEE Trans. on Neural Networks, vol. 4, pp. 570-578, July 1993.

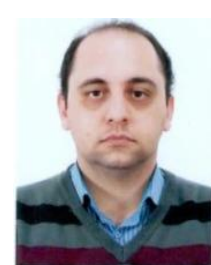

Hamed Alipour-Banaei was born in Tehran, Iran. He received his $\mathrm{PhD}$ degree in electronic engineering from University of Tabriz, Iran. His current research is optical communication networks and photonic based communication systems. 